\title{
An Adaptive Buffer tradeoff, energy-aware Congestion Control protocol in WSN
}

\author{
M. Sri Lakshmi ${ }^{\text {a }}$ Dr.J. Keziya Rani ${ }^{\text {b }}$ \\ ${ }^{a}$ Research Scholar, Department of Computer Science \& Technology, S.K University, Anantapuramu. \\ ${ }^{\mathrm{b}}$ Assistant Professor, Department of Computer Science \& Technology, S.K University, Anantapuramu.
}

Article History: Received: 10 November 2020; Revised 12 January 2021 Accepted: 27 January 2021; Published online: 5 April 2021

\begin{abstract}
In a Wireless sensor network, network lifetime plays a vital role, wherein regular communication and sensor nodes are positioned at different points. Nodes energy depletion may lead to communication interruption due to unlimited data flow from one point to another; for adequate communication, Nodes energy should be maximized by arranging cutting-edge techniques such as adaptive buffer switching and congestion control significant role. When the incoming data is more wideranging than available resources, a congestion situation arises. It results in energy consumption, loss of packets, buffer overflow, and raises end-to-end delay. In this paper, adaptive buffer switching and Congestion Control management are done effectively. Simultaneously, congestion detects based on residual energy, residual buffer space, and sensor nodes conviction level. This methodology shows based on the evaluation of cost, which selects main and spare buffers adaptively. Dynamic buffer switching and swapping are used to enhance the outcome of congestion. Result of the ABETCC approach is compared with the protocol like TCEER and TFCC compared to the data loss ratio and energy consumption
\end{abstract}

Keywords: Congestion, Congestion detection, Congestion control, Sensor nodes, Buffer switching, Buffer swapping, WMSN

\section{Introduction}

Wireless sensor network (WSN) node points can sense physical phenomena [1] in a hostile environment. SN nodes suffer from the energy depletion, and no service restoration. In that situation, there is a need for an energyefficient protocol that improves the network's lifetime. Recently sensor networks are arranged in numerous applications on various pastures such as agriculture, soldierly, green engineering and medical, Etc.

The Wireless Multimedia Sensor Network (WMSN) is a distributed wireless system that, through observing across multiple media, cooperates with the physical world. The endless and vast amount of data transmission from the source to the destination [2] is needed by many WMSN applications.

A vast amount of data and continuous flow of data requires additional resources. Hence congestion occurs in the network if such a situation arises [3]. It results in queuing delay, packet loss, and increase network overhead. Congestion control is a vital task in managing such type of problems. [4].

The contribution of this paper is presented by proposing two efficient approaches to manage network congestion. The goal of the first approach is to decrease the traffic rate and the second method is to ensure efficient control of resources. The proposed ABETCC protocol manages the broad and continuous flow of data to the destination from the source. This protocol relies on the confidence level of the sensor node, remaining buffer space, and remaining energy. In WMSNs, this protocol is specifically intended for efficient buffer management.

This paper is prearranged as tracks- Related Work presented in Section II. Proposed model presented in Section III. Section IV presents the performance evaluation and finally section V concludes the concussion and future scope.

\section{Related Work}

In WSN, congestion management is difficult to assess where congestion has taken place in the network there are so many methods for managing congestion have been identified. In WSN, there are few literatures presented on congestion controlling to lead network lifetime.

The PASCCC [11] defines the network access scheme using novel nodes [17]. It is used in the detection of fires, home automation, and interrelated processes. Points are defined when distinguishing the node roots. Arriving packages are prioritized by selecting one with the least desired arrival time (at first glance, packages in the system seem to be prioritized) low-priority packets [18] are always dropped when the network is congested 
The TFCC [6] proposed in WSN for a trust-based congestion control scheme [6] dynamically, the traffic flow is modified based on priority. The faulty nodes blocked during data communication were also established on the basis of the confidence assessment, through which packet transmission congestion [19] was reduced. Based on the two-stage fuzzy inference scheme, the trust value of the nodes is assessed. TFCC utilizes a system for link-state routing.

The author [13] suggested an approach called TCEER which is suitable for energy-efficient routing for corporate bottleneck warnings. In addition to several safety attacks from spooky points, the envisaged device safeguards sensor points. The optimal method is selected using a fuzzy logic supervisor founded on the node possible value, measured from confidence [20], residual energy, congestion level, and node distance. The source point selects an energy efficient and substantially potential hop neighbour. A hop through the hop routing system is used by TCEER.

The reduction in the data transfer rate is carried out by most congestion control techniques. Rather than offering an exact solution, these approaches would avoid congestion. Some other methods work to prevent congestion by effectively utilizing the resources available. [21].

Protocols such as TFCC[6] and TCEER[13] focus on data rate reduction and efficient use of resources during congestion. Both mechanisms are capable of managing congestion, but time and delay parameters are influenced by performance [22].

\section{Proposed Network Model}

\section{Adaptive Buffer tradeoff, energy-aware and Trust-based Congestion Control protocol (ABETCC)}

In WMSNs, for a broad and continuous flow of data this protocol relies on the sensor node's remaining resources, buffer, and trust level. It is designed to handle buffers effectively in WMSNs. A Network Topology Development for original implementation is discussed in the following subsection. The second sub section explains the measurement of confidence for neighborhoods. Stable data packet generation, routing and load balancing, and proposed device implementation are addressed in the third sub-section. The estimation of the cost function is seen in the fourth segment. Congestions are defined by different congestion indicators. The final section sets up an effective strategy for reducing congestion, where packet loss decreases.

\section{Network Topology Creation}

The stepwise network model is designed in the following manner.

For determining its efficiency, the topology of a network is vital. Network topology is organized in the network, with a physical or logical definition of connections and nodes.

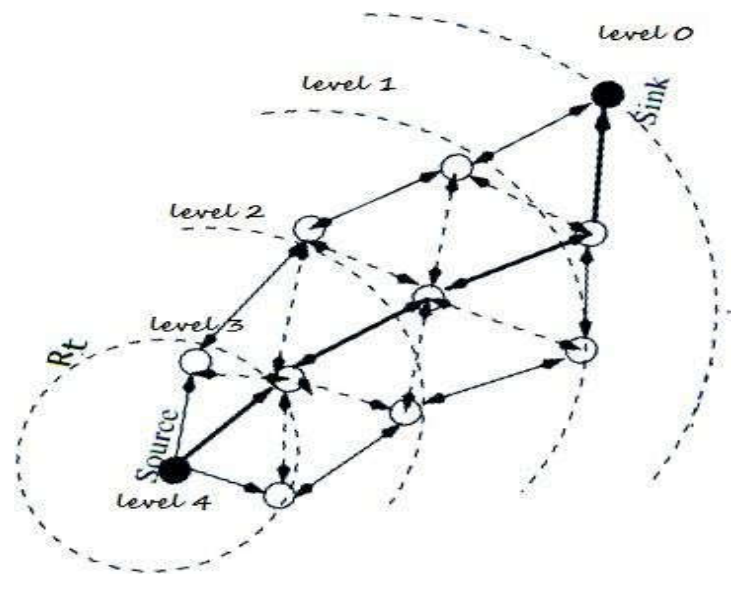

Figure 1 Topology creation of WSN

The deployment of the sensor nodes when the experiment is started could be random. The primary base station sends the ' HAI SRILAKSHMI ' message, which starts the hierarchical topology development. The HAI SRILAKSHMI ' message received by the sensor nodes, which preceedes the topology formation, and increases the hop count as well as the topology. - HAI SRILAKSHMI\| consists of the node ID, the amount of energy in the path, and how many hops (more precisely, how many are left to reach the base station) is left. For sensor nodes with a value of 0 , that is, we have a base station; for those with a greater number, a base station; for more than one, we have more than one base station this method repeats as long as there are additional nodes to 
expand the tree. When each node received full information of levels, positions, prior energy, and buffers, all the neighbors on the current structure were also completed, they each node started the topology expansion phase Expanding the information in Figure 1

\section{Trust evaluation}

Existing congestion control protocols solve the severe congestion problem using unilateral congestion control approach [4][5][6]. That is the use of either traffic control or resource control strategy-for instance, an inverse relationship between energy and throughput in these approaches.

$$
E=\frac{1}{T P}
$$

Where E=Energy

$$
\mathrm{TP}=\text { throughput }
$$

Good energy and throughput performance cannot be achieved through this unilateral approach.

A number of different factors are taken into consideration in the calculation of the trust model; it gives an accurate estimate of trust. a DTEM (Direct Trust Evaluation Model) that has the ability to change both the weight of direct and indirect trust. This is the extent of how one's own self-efficacy and the ability to adapt to different environments.

- The trust score takes into consideration the actual trust level as well as collateral trust to ensure that enables trust ability in trust attacks. For wireless sensor networks, we present a novel trust evaluation model.

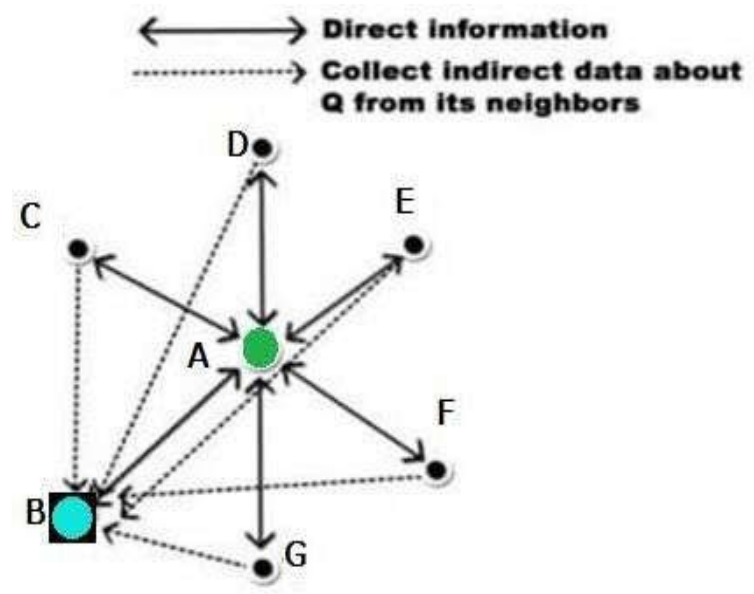

Figure:2 - Direct and Indirect Approach

\section{Trust evaluations}

The Direct Trust (DT) of a node $B$ arranged a node $A$ is based on recommendation trust and corresponding Weight value. The results of direct trust evaluation between the relay node $\quad A$ and supervised node can be estimated as:

$$
\text { DTrust }=\sum_{m=1} w_{m} * t m_{m}^{B A}
$$

Where $t m_{m}^{B A}$ Trust metric of a node $B$ on a node $A$.

Indirect trust

IT of a node
$B A$$\sum_{y}$ on a node $A$ created on recurrent trust metrics is deliberate as follows,

$$
I T=\frac{-}{n} \sum_{x=1}\left(W_{B N x} * T^{N x, B}\right)
$$


Where

$W_{B N x}$ Is the weight for the recommendation made by Xth Neighbor

$T^{N x, B}$ Does neighbor node Nx give trust about node $A$

$\mathrm{X}$ denotes highest trustworthy neighbor,

y denotes trustworthy middle neighbor,

$\mathrm{z}$ denotes the lowermost reliable neighbor.

The TT of a node $B$ on a node $A$ established on recurrent trust metrics is deliberate as follows.

$$
\mathrm{T}^{\mathrm{B}, \mathrm{A}}=\mathrm{W}_{\mathrm{D}} * \mathrm{DT}^{\mathrm{B}, \mathrm{A}}+\mathrm{W}_{\mathrm{I}}^{*} \mathrm{IT}^{\mathrm{B}, \mathrm{A}}
$$

Where

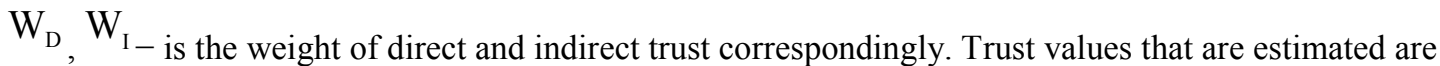
comprised in the neighbor table.

\section{Routing and Load balancing}

The emphasis on energy consumption is on Wireless Sensor Networks (WSN). Energy is a severely scarce resource for WSNs. This restriction limits the activity of the sensor nodes and somehow compromises the efficiency and an operation can involve all of the long-term or many networks at once in some manner or another, depending on the complexity of the network design. In order to increase network efficiency and ensure node life expectancy, we make our protocols customizable for nodes, matching the energy requirements of the nodes to the protocol.

In wireless sensor networks, high node density and source-to-sink communication patterns lead to congestion and packet losses. An improved metric is used to detect congestion, considering the channel condition and queue length at the one-hop neighborhood. For avoiding congestion and bottleneck nodes, each node dynamically adjusts the for load balancing purposes, the transmitter offers each one-hop neighbor a different transmission rate and balance ratio.

\section{For Routing:}

For wireless communication, the two-dimensional (or free-space) model or radio model are typically used. The free Space model is applied when the communication distance $\mathrm{d}$ is less than or equal to the threshold $\mathrm{d} 0$. Furthermore, a multiple fading model is utilized. For the Energy radio, the k-bit message takes d units of energy to travel from one location to another.

$$
\begin{aligned}
& \mathrm{E}_{\mathrm{TX}}=\mathrm{E}_{\text {ele }}(\mathrm{k}, \mathrm{d})+\mathrm{E}_{\mathrm{amp}}(\mathrm{k}, \mathrm{d}) \\
& =\mathrm{k} * \mathrm{E}_{\text {ele }}+\mathrm{k} * \in_{\mathrm{fs}}(\mathrm{d}<\mathrm{d} 0) \\
& =\mathrm{k} * \mathrm{E}_{\text {ele }}+\mathrm{k} * \in_{\mathrm{mp}}(\mathrm{d}>=\mathrm{d} 0)
\end{aligned}
$$

Energy received is calculated as follows.

$$
\mathrm{E}_{\mathrm{RX}}=\mathrm{k} * \mathrm{E}_{\mathrm{ele}}
$$

Current energy is calculated as

$$
\mathrm{E}_{\mathrm{B}}=\mathrm{Ei}-\left\{\mathrm{E}_{\mathrm{TX}}(\mathrm{k}, \mathrm{d})+\mathrm{E}_{\mathrm{RX}}(\mathrm{k})\right\}
$$

Where

Ei represents as primary energy of the SN (assessed throughout Network topology formation)

$\mathrm{E}_{\mathrm{TX}}(\mathrm{k}, \mathrm{d})$ represents for distance $\mathrm{d}$ what is the energy consumed while transferring the $\mathrm{K}$ bits of data. 
$\mathrm{E}_{\mathrm{RX}}(\mathrm{k})$ represents for getting $\mathrm{K}$ bits of data what energy consumed.

The Total Energy of the SN is calculated as follows.

$\mathrm{E}_{\mathrm{T}}=\mathrm{ET}_{\mathrm{X}}(\mathrm{k}, \mathrm{d})+\mathrm{ER}_{\mathrm{X}}(\mathrm{k})+$ Eproc + Esense

The node P's remaining energy is determined as follows.

$R E(P)=\frac{E_{P}}{E_{T}}$

$E_{P}$ stands for the SN currentenergy

$\mathrm{E}_{\mathrm{T}}$ stands for the $\mathrm{SN}$ total energy.

Based on the current buffer status and the estimated sum of packets communicated from node $\mathrm{P}$ to its highest level, a node's remaining buffer is determined. The node P's remaining buffer is determined as follows.

$\mathrm{RB}(\mathrm{B})=\mathrm{B}_{\mathrm{i}}-\sum_{N=1}^{N} k_{N}$

Where

$\mathrm{B}_{\mathrm{i}}$ denotes the buffer status at node.

$\mathrm{K}_{\mathrm{N}}$ where $\mathrm{N}$ is the amount of the packets communicated from $\mathrm{P}$ to its higher level.

\section{Proposed System Implementation}

The proposed adaptive congestion control protocol for Buffer switching, Energy-aware and Trust-based Congestion Control (ABETCC) is intended to manage a broad The movement of data from the source to the destination is an essential component of a supply chain processes. In a dispute, it all depends on how much energy is available, how much is left in the system, and on the suspect's level of confidence. The first thing it is made for is buffer efficiency in WSNs.

Proposed model: Flow Diagram.

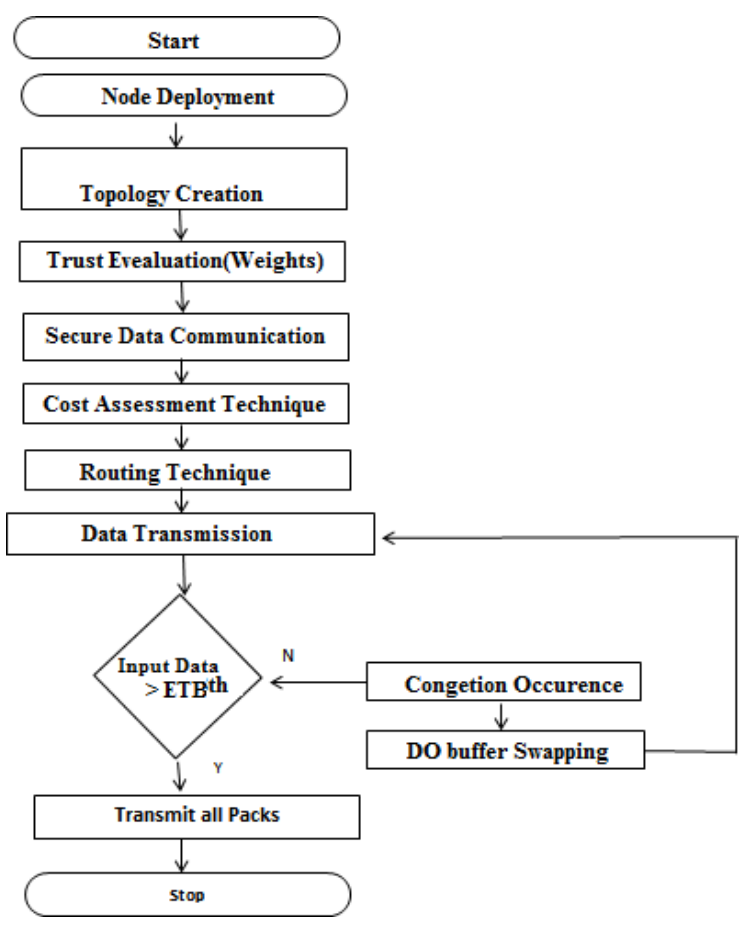

Figure 3 - Basics steps of ABETCC Protocol 


\section{Algorithm 1.1: Buffer swapping}

1. Input: Incoming Data( $\left.D_{i}\right)$

Current Buffer( $\left.B_{c}\right)$

Spare Buffer (Bs)

2. System Parameters

Node parameters : $\mathrm{R}_{\mathrm{b}}$ (Buffer Rate);

Delay $=0$;

Buffer Size( $\left(B_{s}\right)$;

3. Initialization

$E T B^{\text {th }}=1$;

4.Start to send data from node 1 to Node 2

delay $=0$

Speed of data sending is high

$\mathrm{D}_{\mathrm{i}}$ : Connect to $\left(\mathrm{B}_{\mathrm{c}}\right)$

5. Step 1:

if( $\left(\mathrm{DR}_{\mathrm{i}} \geq \mathrm{ETH}^{\text {th }}\right)$

then Do

Congetion detected =true;

alert signal to (Buffer Swapping or Buffer Switching)

Increase $\mathrm{D}_{\mathrm{i}}$ packetization delay by 3 ;

else

do

Congetion detection $=$ False;

$\mathrm{D}_{\mathrm{i}} \cdot \mathrm{B}_{\mathrm{c}}++$

continues the data transmission

goto step 1 ;

The spare buffer is triggered by alarm signals; the current buffer assumes responsibility. The main buffer is now going to the sleep mode for energy conservation. The process is called buffer refinement from the primary buffer to the secondary buffer.

Buffer tradeoff is described in Algorithm 1.1.

\section{Buffer swapping:}

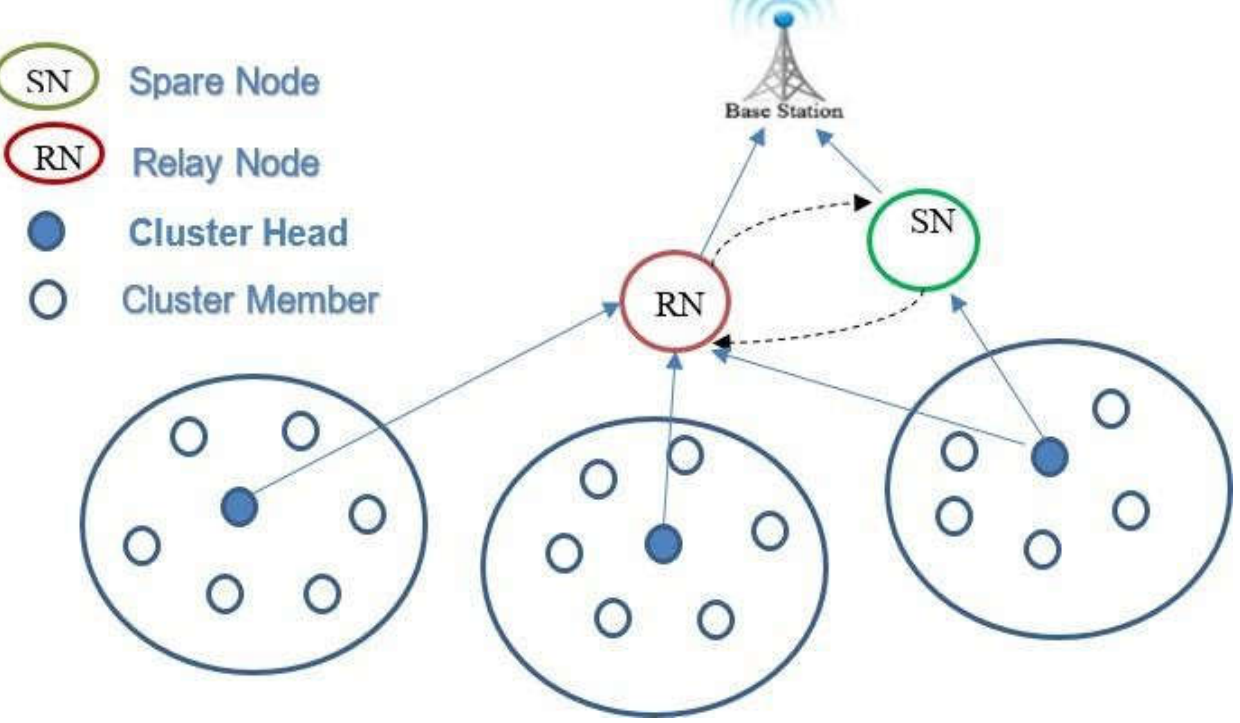

Figure 4 -Buffer tradeoff 
The Buffer swapping technique has been introduced in the proposed system to show effective buffer management in WMSNs. In Fig 4, it is represented that the data is transferred from the Cluster Heads $(\mathrm{CH})$ to the relay node. From the Relay node $(\mathrm{RN})$, the data is transferred to the base station. If the data receiving from $\mathrm{CH}$ is extensive and the RN does not have enough buffer space, then RN will send the signals to all the cluster heads to transfer the data to the Spare Node(SN). Then the $\mathrm{CH}$ will divert transmitting the data to the spare node. When the Buffer of RN gets empty, the SP swaps the data to RN Buffer.

\section{Performance evaluation}

The performance evaluation of the ABETCC is used to conduct the simulation compared to - TFCC and TCEER $\|$ by NS: 2.30 . Table 1 lists the necessary parameters. Area is $500 X 500 \mathrm{~m}$, sensor points are installed. The lower left part of the field begins any transmission and BS is located in the top left of the region. Communication range is $40 \mathrm{~m}$ and sensor range is $50 \mathrm{~m}$ remaining parameter are shown in table 1 .

Table: 1 - Simulation parameters

\begin{tabular}{|l|l|}
\hline Area of WSN & 500 X 500m \\
\hline Number of nodes & 600 \\
\hline Protocol Used & AODV \\
\hline $\begin{array}{l}\text { Range for } \\
\text { communication }\end{array}$ & $40 \mathrm{~m}$ \\
\hline Range for sensing & $50 \mathrm{~m}$ \\
\hline Length of the packet & 60 bytes \\
\hline Length of the Buffer & 65 packets \\
\hline Initial Energy & $65 \mathrm{~J}$ \\
\hline Data rate & $200 \mathrm{kbps}$ \\
\hline Eelem & $60 \mathrm{nj} / \mathrm{bit}$ \\
\hline Lamps & $10 \mathrm{pJ} / \mathrm{bit} / \mathrm{m}^{2}$ \\
\hline E sensing & $0.090 \mathrm{~J} / \mathrm{s}$ \\
\hline
\end{tabular}

\section{Proposed protocol performance metrics:}

1. The amount of energy that we left

2. The number of packets lost in a critical condition

3. Shows the energy consumption of a node at a given time.

4. Displays Node measurements as well as typical energy

consumption. 5.Typical energy usage and data rate

6. Packet loss when a large number of nodes are involved in packet transmission.

The remaining energy level of many protocols at the time of packet transmission, as shown in Table 2, is the first efficiency metric. When faced with a crisis, the remaining energy level is crucial. Fig. 5 demonstrates the efficiency in energy conservation of the proposed protocol ABETCC over the current protocols TFCC and TCEER.

Table 2 - Remaining Energy of various Protocols

\begin{tabular}{|c|l|l|l|}
\hline \multirow{2}{*}{ Node-ID } & \multicolumn{3}{|c|}{ Remaining Energy } \\
\cline { 2 - 4 } & \multicolumn{1}{|c|}{ TFCC } & TCEER & ABETCC \\
\hline 10 & 0.76 & 0.8 & 0.84 \\
\hline 20 & 0.69 & 0.76 & 0.81 \\
\hline 30 & 0.67 & 0.7 & 0.75 \\
\hline 40 & 0.63 & 0.65 & 0.71 \\
\hline 50 & 0.57 & 0.59 & 0.64 \\
\hline 60 & 0.55 & 0.57 & 0.62 \\
\hline 70 & 0.53 & 0.55 & 0.60 \\
\hline 80 & 0.48 & 0.50 & 0.58 \\
\hline 90 & 0.45 & 0.47 & 0.55 \\
\hline 100 & 0.41 & 0.43 & 0.51 \\
\hline & & & \\
\hline
\end{tabular}




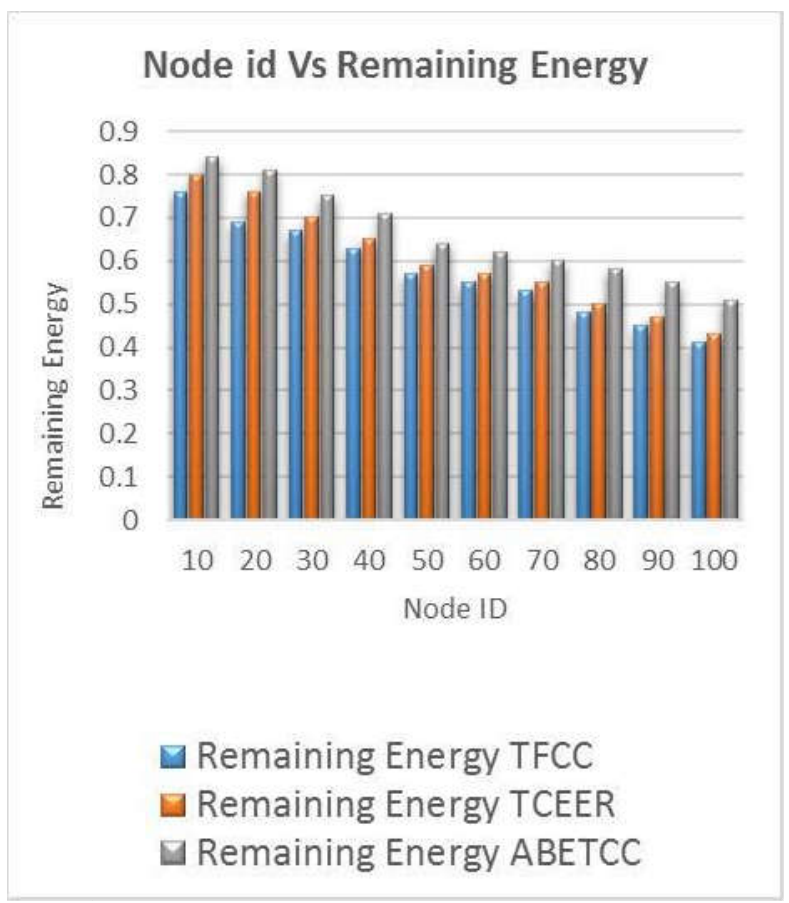

Fig 5: Node Vs. remaining energy

The number of packet loss during critical condition constitutes the second performance metric. Table 3 shows that The Congestion control algorithm decreases the number of packet loss and performance of the network degrades. ABETCC method shows better results compared to existing congestion control protocols TCEER and TFCC.

Table 3: Counting the number of packets lost during a crucial condition

\begin{tabular}{|l|l|l|}
\hline Protocol & Time & Packet loss \\
\hline TFCC & 3 & 770 \\
\hline TCEER & 3 & 700 \\
\hline ABETCC & 3 & 560 \\
\hline
\end{tabular}

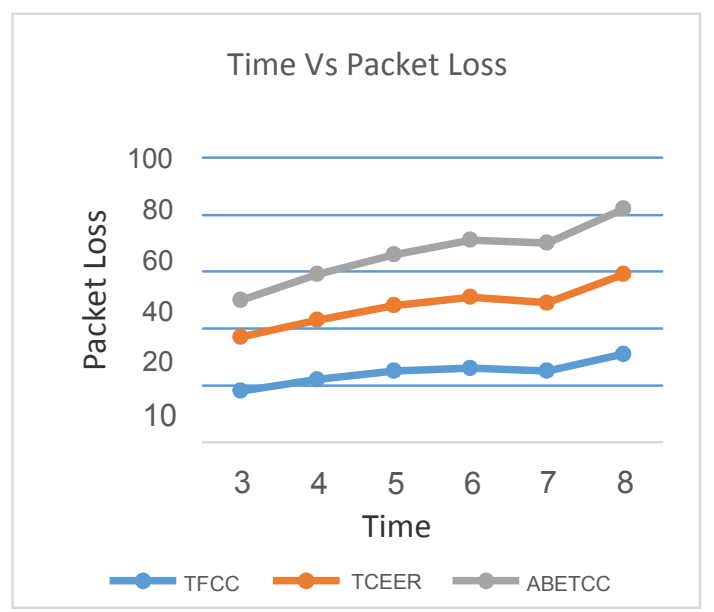

Fig 6: Time Vs. packet loss 
The node's energy consumption at a given time is the third efficiency metric shown in Table 4. Energy is typically used when a packet is received or a packet is sent or an incoming packet is processed and data is sensed on a network. Compared to TFCC and TCEER, the proposed protocol, ABETCC, uses the least amount of resources. The time versus energy consumption result is shown in Fig 7.

Table 4: Node Energy at a given point of time.

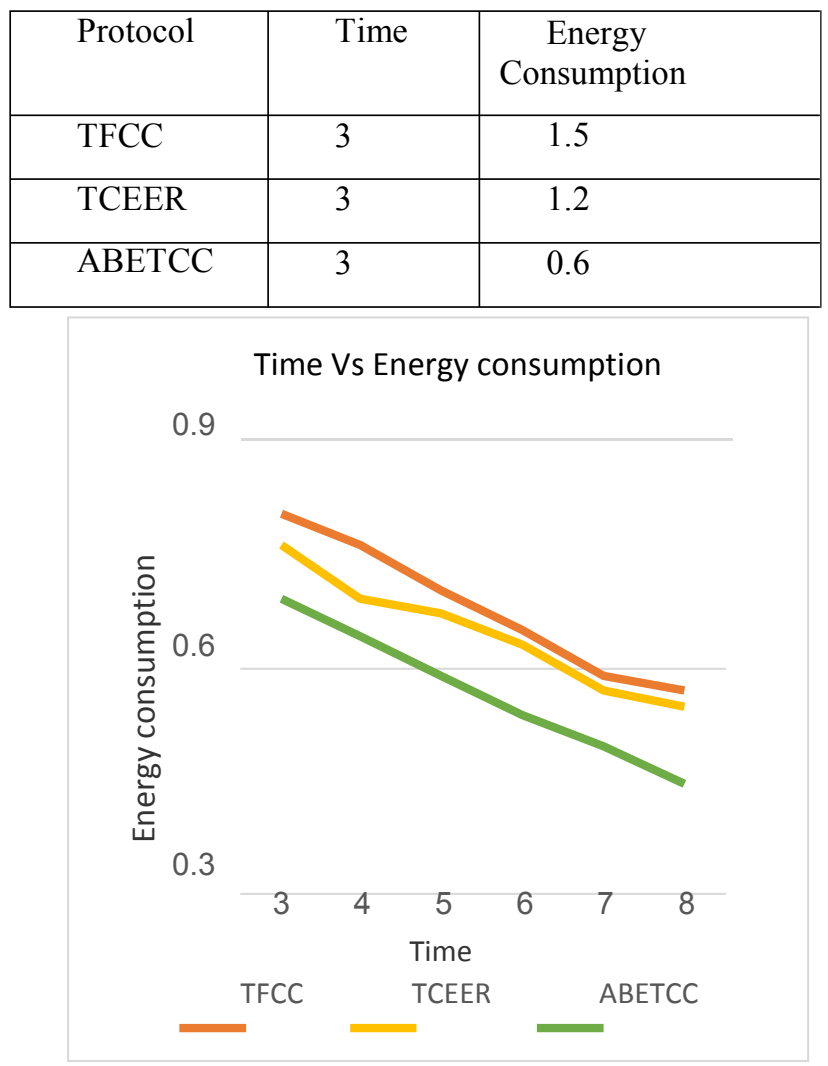

Fig 7: Time Vs. Energy consumption

Table 5 shows the typical energy consumption of nodes, as well as data rate vs typical energy consumption. The fourth and fifth output metrics are included. As the number of nodes increases, more energy and buffer space is absorbed by the network, contributing to its efficiency degradation. With the increased number of nodes and data rates, as shown in Figs 8 and 9, ABETCC preserves additional energy than -TCEER and TFCC.

Table 5: Data rate vs. typical energy consumption

\begin{tabular}{|c|c|c|c|c|}
\hline \multirow[b]{2}{*}{ Nodes } & \multirow{2}{*}{$\begin{array}{l}\text { Data } \\
\text { rate } \\
(B / S)\end{array}$} & \multicolumn{3}{|c|}{ Typical Energy Consumption } \\
\hline & & TFCC & TCEER & ABETCC \\
\hline 100 & 10 & 0.8 & 0.76 & 0.69 \\
\hline 200 & 20 & 0.76 & 0.69 & 0.64 \\
\hline 300 & 30 & 0.7 & 0.67 & 0.59 \\
\hline 400 & 40 & 0.65 & 0.63 & 0.54 \\
\hline 500 & 50 & 0.59 & 0.57 & 0.5 \\
\hline 600 & 60 & 0.57 & 0.55 & 0.45 \\
\hline
\end{tabular}




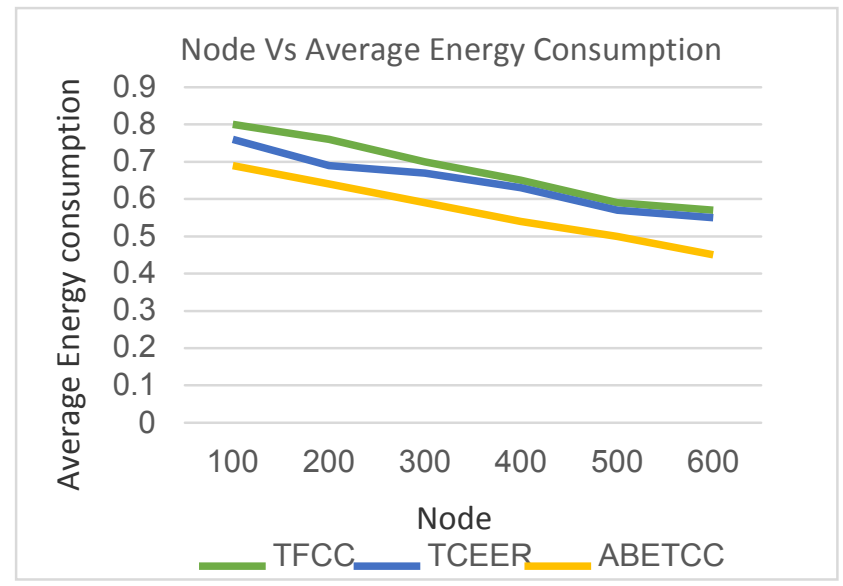

Fig 8: Nodes vs. typical energy Consumption

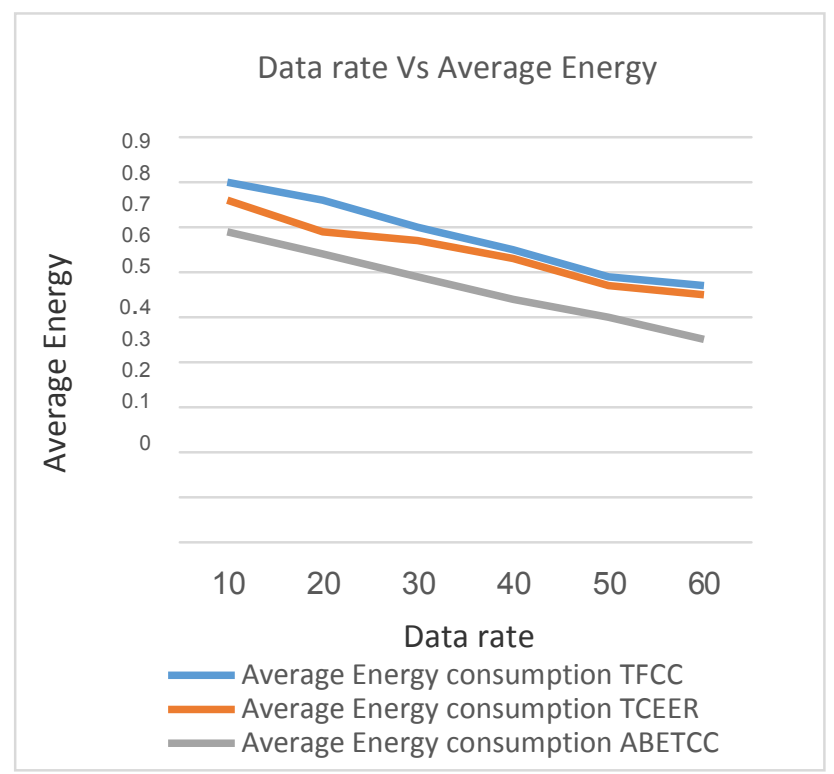

Fig 9: Data rate vs. typical energy Consumption

\section{Conclusion and Future Work}

To avoid congestion in WMSNS Trust-based Congestion Control, residual Buffer, residual energy has been used to design the proposed protocol. It is demonstrated that for the initial WSN, hierarchical topology is established. Trust metrics are evaluated using the neighbor node's trust value. Creation of secure data packet, Routing, and Load balancing presented with mathematical evaluation. The proposed model classifies into two types: main and spare. The data packet would be handled by the Primary Buffer if there was no network congestion. During data transmission, buffer switching is done if there is congestion. By allowing the spare Buffer to manage congested regions quite effectively, the primary Buffer produces a wake-up signal. The suggested protocol in next- hop selection increases the Buffer's residual energy level by 19.26 percent more than TCEER, 23.61 percent more than TFCC. In the proposed protocol, the packet loss is $29.16 \%$ lower than TFCC and $21.62 \%$ lower than TCEER. In developing energy-efficient trust-based congestion management protocols, complex shifts in nodes and their neighboring positions can be considered in the future. 


\section{References}

1. K. Sumathi1 \& P. Pandiaraja(2019), Dynamic alternate buffer switching and congestion control in wireless multimedia sensor networks, Springer Science Business Media, LLC, part of Springer Nature.

2. R Jiang, H Zhou, H Wang, SSGe (2019) Maximum entropy searching. CAAI Transactions on Intelligence Technology 4(1):1-8

3. Tang Z, Gong M (2019) Adaptive multi factorial particle swarm optimization. CAAI Transactions on Intelligence Technology 4(1):37-46

4. Lalwani S, Sharma H, Verma A, Kumar R (2019) Efficient discrete firefly algorithm for Ctrie based caching of multiple sequence alignment on optimally scheduled parallel machines. CAAI Transactions on Intelligence Technology 4(2):92-100

5. Wan C-Y, Eisenman SB, Campbell AT (2003) CODA: congestion detection and avoidance in sensor networks. SenSys'03 Proceedings of the 1st international conference on Embedded networked sensor systems 266-279

6. Chakraborty A et al. (2013) A trust-based fuzzy algorithm for congestion control in wireless multimedia sensor networks (TFCC). In: Presented in the proceedings of the IEEE international conference on informatics, Electronics \& Vision, Dhaka, Bangladesh

7. Wan CY etal (2002)PSFQ: a reliable transport protocol for wireless sensor networks. Presented in the ACM international workshop proceedings on wireless sensor networks and applications, Atlanta. http://wsn.cerist.dz/wp-content/uploads/2015/03/Submission00123-2013-.pdf

8. Sonmez C, IncelOD, Sinan Isik MYD, ErsoyC (2014)Fuzzy-based congestion control for wireless multimedia sensor networks. EURASIP Journal on Wireless Communications and Networking 2014(1):63

9. Jan MA, Nanda P, He X, Liu RP (2014) PASCCC: Priority-based application-specific congestion control clustering protocol. Computer networks 74:92-102

10. Basaran C, Kang K-D and Suzer MH Hop-by-hop congestion control and load balancing in wireless sensor networks. IEEE Local Computer network conference448-455. https://doi.org/10.1109/

11. Chakraborty A et al. (2015) Trust integrated congestion aware energy-efficient Routing for wireless multimedia sensor networks (TCEER). Journal of computing and information technology 2015(2):95109

12. John Paul Walters, -Wireless sensor network security: a surveyll security in distributed, grid, and pervasive computing Yang Xiao, (Eds.) pp 2006 Auerbach Publications, CRC Press. https://www.cse.buffalo.edu/ vipin / book_Chapters/2007/2007_2.pdf

13. Kafi, M.A., Djenouri, D., Ben-Othman, J., Badache, N. Congestion control protocols in wireless sensor networks: a survey. IEEE Communications Surveys \&Tutorials, accepted for publication 2013.

14. Sumathi K, Venkatesan M, A Survey on Congestion Control in Wireless Sensor Networks -International Journal of Computer Applications (0975-8887), 2016, 147(6):6-11

15. Bala Anand, M. Karthikeyan, N.\& Karthik, S."Designing a framework for communal software: based on the assessment using relation modelling," Int JParallelProg(2018). https://doi.org/10.1007/s10766018 0598-2 M.BalaAnand, S.Sankari, R. Sowmipriya, S. Sivaranjani "Identifying fake user's in social networks verbal behavior," International Journal of Technology and Engineering System 7(2):157-161

16. Maram B, Gnanasekar JM, Manogaran G, et al. (2018) Intelligent security algorithm for UNICODE data privacy and security in IoT. SOCA 13:3-15

17. BalaAnand M, Karthikeyan N, Karthick S, Sivaparthipan CB (2018) Demonetization: a visual exploration and pattern identification of people opinion on tweets. 2018 international conference on soft-computing and network security, pp1-7https://doi.org/10. 1109/ICSNS.2018.8573616

18. Anupriya K, Gayathri R, Bala anand M, Sivaparthipan CBE shoppings cam identification using machine learning, 2018 international conference on soft-computing and network security, 1-7

19. Sivaparthipan CB, Karthikeyan N, KarthikS(2018) Designing statistical assessment on health care information system for diabetics analysis using big data. Multimedia Tools Appl.

20. Solomon Z, Sivaparthipan CB, Punitha P, Bala Anand M, Karthikeyan N (2018) Certain investigation on power preservation in sensor networks. In: 2018international conference on soft-computing and network security (ICSNS), Coimbatore. IEEE Xplore, 13 December 2018

21. Bala Anand M, Karthikeyan N, S Karthik CB (2017) Sivaparthipan "a survey on BigData with various V's on comparison of apache hadoop and apache spark." Advances in Natural and Applied Sciences. 